\title{
Planets Born from Dying Stars
}

\author{
One explanation of a new analysis of dying binary-star systems is that \\ planets might be forming in the "ashes" of stars.
}

By Sarah Wells

S cientists studying the births of planets typically spend their time analyzing the rings of dust, known as protoplanetary disks, that form around freshly burning stars. From this dust, they have seen evidence of matter coming together to form protoplanets that, like Earth, orbit a single star. Now, Jacques Kluska and Hans Van Winckel from KU Leuven, Belgium, and colleagues report observations that indicate that there might exist another route for stars to birth planets. This finding could help scientists better understand how common planets are in the Universe.

Kluska, Van Winckel, and colleagues consider a type of evolved binary-star system known as a post-asymptotic giant branch (post-AGB) binary. In this binary system, when one of the stars dies, a disk of cosmic building materials forms around the dead star-and the star that's still alive. The makeup of such a disk

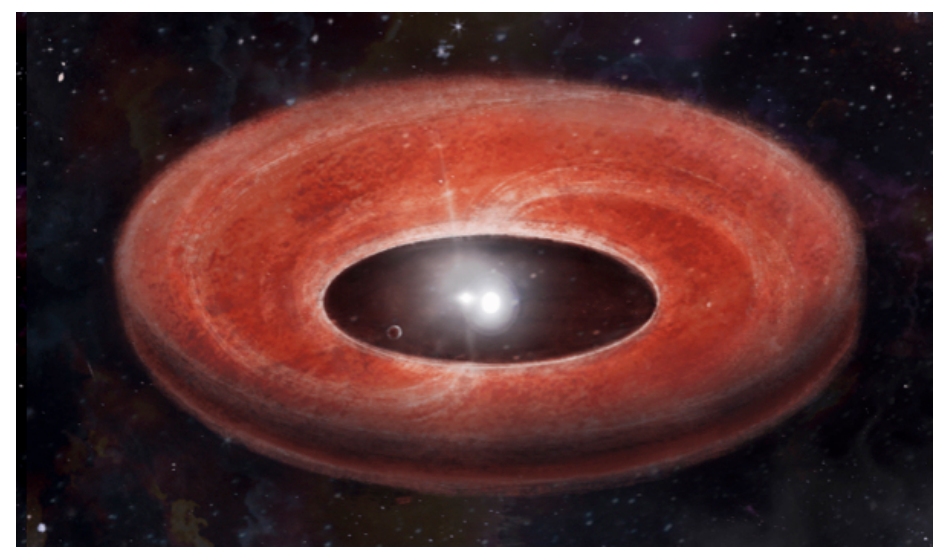

Researchers have discovered binary-star systems that have an unusual cavity at the center of their surrounding disk of gas and matter (right). This cavity might be evidence that planets form in these systems.

Credit: N. Stecki has been shown to be very similar to that of a protoplanetary disk, which is known to birth planets. Van Winckel says that this similarity led him and his colleagues to decide to investigate the dust more deeply. "You could say that nature showed us the way," he says.

In their study, Kluska, Van Winckel, and colleagues looked at 85 post-AGB binaries. They studied archival data for each system. They also collected new spectrograph data using the Mercator telescope in Spain. The team analyzed the infrared emission from each post-AGB binary to characterize the composition of its dust and to categorize the morphology of its disk. They then compared data from all the post-AGB binaries. They found that $10 \%$ appear to house a matter cavity at their centers. This cavity depletes iron and other refractory elements from the dying star's surface.

To explain this element depletion, the team explored several causes, including the presence in the post-AGB binary of a star that is naturally low in the missing elements. But ultimately, they zeroed in on it arising from the existence of a newly formed planet. The presence of a young planet is the "best explanation we came up with," Kluska says.

This finding opens a "new vista" for the study of the disks around post-AGB binaries, says Yanqin Wu, a professor of theoretical astrophysics at the University of Toronto, whose work focuses on the study of protoplanetary disks. "The planet hypothesis is titillating," she says. "If new planets do form in the center of the disks around dying stars, they will be like a phoenix rising out of the ashes." However, while Wu is intrigued by the team's explanation for their findings, she is not convinced that a planet is the only solution to the observed puzzle and says "other physical explanations could connect the two dots." 
To find out if their planet hypothesis is correct, Kluska, Van Winckel, and their colleagues say that they plan to collect and analyze further data. Specifically, the team will use high-spatial-resolution images from observatories such as the Very Large Telescope and the Atacama Large

Millimeter/submillimeter Array, both in Chile, to study the structure of the post-AGB binaries in more detail. If the planet hypothesis is proven correct, post-AGB binary systems could serve as a new laboratory to test models of planet formation, Kluska says.

Sarah Wells is a freelance science journalist based in Boston. 